\title{
Germline HNPCC gene variants have little influence on the risk for sporadic colorectal
}

\section{cancer}

\author{
I P M Tomlinson, N E Beck, T Homfray, C J Harocopos, W F Bodmer
}

\begin{abstract}
Hereditary non-polyposis colorectal cancer (HNPCC) is a syndrome of inherited bowel and other cancers that has been said to account for up to $15 \%$ of all colorectal carcinomas (CRCs). HNPCC can now be diagnosed at the molecular level by detecting germline mutations in genes involved in mismatch repair. A current problem is to determine the prevalence of HNPCC mutations in colon cancer patients with limited or no family history, especially in cases of early onset. We have identified 50 cases of non-polyposis colorectal cancer without a family history of CRC or any other HNPCC cancer, who presented under the age of 45 years. Germline HNPCC variants (at the hMSH2 or hMLH1 loci) were detected in a small minority of cases $(6 \%)$. The variants that we have found may be new or low penetrance mutations, or even polymorphisms. It remains possible that some of our sample have an inherited predisposition to CRC that is not caused by HNPCC mutations or by known polyposis syndromes. Our data suggest that most HNPCC mutations occur in families and have high or moderate penetrance. New or low penetrance HNPCC mutations probably do not contribute significantly to the risk of colorectal cancer in the general population and probably account for much fewer than $15 \%$ of all CRCs. Our results question whether mass population genetic screening programmes are worthwhile for diseases such as HNPCC using current technology. ( $\mathcal{F}$ Med Genet 1997;34:39-42)
\end{abstract}

Cancer Genetics Laboratory, Imperial Cancer Research Fund, 44 Lincoln's Inn Fields, London WC2A

IPX, UK

NE Beck

T Homfray

W F Bodmer

Colorectal Cancer

Unit, Imperial Cancer Research Fund, St Mark's and Northwick Park Hospitals Trust, Watford Road, Harrow, Middlesex HA1 3UJ, UK

C J Harocopos

Correspondence to: Dr Bodmer.

Received 13 May 1996 Revised version accepted for publication 29 August 1996
Keywords: HNPCC; early onset sporadic colon cancer.

Hereditary non-polyposis colorectal cancer (HNPCC) is a syndrome of inherited bowel cancer that accounts in some studies for 5 to $15 \%$ of all colorectal carcinomas (CRCs). ${ }^{1}$ Colon cancers in HNPCC families tend to be right sided, early onset, mucinous, multiple, and of good stage for stage prognosis. Clinically, HNPCC has been defined according to the "Amsterdam criteria": (1) three or more relatives affected by CRC, one a first degree relative of the other two; (2) two or more generations affected; (3) one or more CRCs presenting before 50 years of age; and (4) exclusion of hereditary polyposis syndromes.

These criteria have been criticised on two grounds. First, they do not include the extracolonic tumours that tend to occur in HNPCC families, particularly cancers of the uterus, ovary, kidney, stomach, skin, and urinary epithelium. Second, the "Amsterdam criteria" may wrongly exclude patients with a more limited family history (owing to factors such as new mutations, reduced penetrance, death before cancer is diagnosed, and incomplete history taking or diagnosis).

Most families with HNPCC are caused by germline mutations in one of four genes (hMSH2, hMLH1, hPMS1, hPMS2) that are involved in DNA mismatch repair. ${ }^{2-5}$ As an alternative to the "Amsterdam criteria", it is now possible to define HNPCC by the presence of a functional germline mutation at an HNPCC locus. Two problems of immediate importance are to determine the prevalence of HNPCC mutations (1) in colon cancer families with relatively late age of onset and (2) in people with colon cancer but without significant family history. In the latter case, whether or not patients with apparently sporadic colon cancer actually have germline HNPCC mutations greatly influences their own risk of metachronous colon cancer and extracolonic cancers, and affects their relatives' risk of cancer. Germline HNPCC mutations are likely to be most frequent in non-familial CRCs which present at an early age, owing either to new mutations or to low penetrance HNPCC alleles, which might contribute to the risk of cancer in all age groups. If, however, germline HNPCC mutations are rare in young colon cancer patients, it suggests that most HNPCC mutations have high penetrance, that most HNPCC occurs within families, and that HNPCC is responsible for fewer CRCs than the $15 \%$ suggested by some studies.

From medical records, we have identified 50 cases of colorectal cancer without family history that presented at $\leq 45$ years of age (median 39, range 15-45) (table 1). The risk of CRC to the first degree relatives of patients with CRC at $\leq 45$ years is about 1 in $10,{ }^{6}$ compared with a population risk of about 1 in 35 , but these data are not strictly comparable with our study. No patient was included in our study if

\begin{tabular}{lc} 
Table 1 & Age distribution of the patients studied \\
\hline Age $(y)$ & No of patients \\
\hline$<20$ & 1 \\
$21-25$ & 1 \\
$26-30$ & 4 \\
$31-35$ & 6 \\
$36-40$ & 22 \\
$41-45$ & 16 \\
\hline
\end{tabular}


Table 2 Clinicopathological features and mutations detected in the early onset cases of colorectal cancer studied

\begin{tabular}{llllllllll}
\hline Case & Age & Site & Stage & Grade & Gene & Exon & Codon & DNA change & Amino acid \\
\hline 78 & 40 & Rectum & B & PD & hMLH1 & 8 & 219 & ATC $\rightarrow$ GTC & Ileu $\rightarrow$ Val \\
JM & 40 & Transverse & B & NR & hMSH2 & 6 & 322 & GGC $\rightarrow$ GAC & Gly $\rightarrow$ Asp \\
YKY & 37 & Sigmoid & C & MD & hMLH1 & 3 & 77 & TGT $\rightarrow$ TAT & Cys $\rightarrow$ Tyr \\
\hline
\end{tabular}

Three germline variants (all missense) are shown. Duke's stage (A, B, or C) is shown. Tumour grade is expressed as well, moderately, or poorly differentiated (WD, MD, PD). NR= not reported.
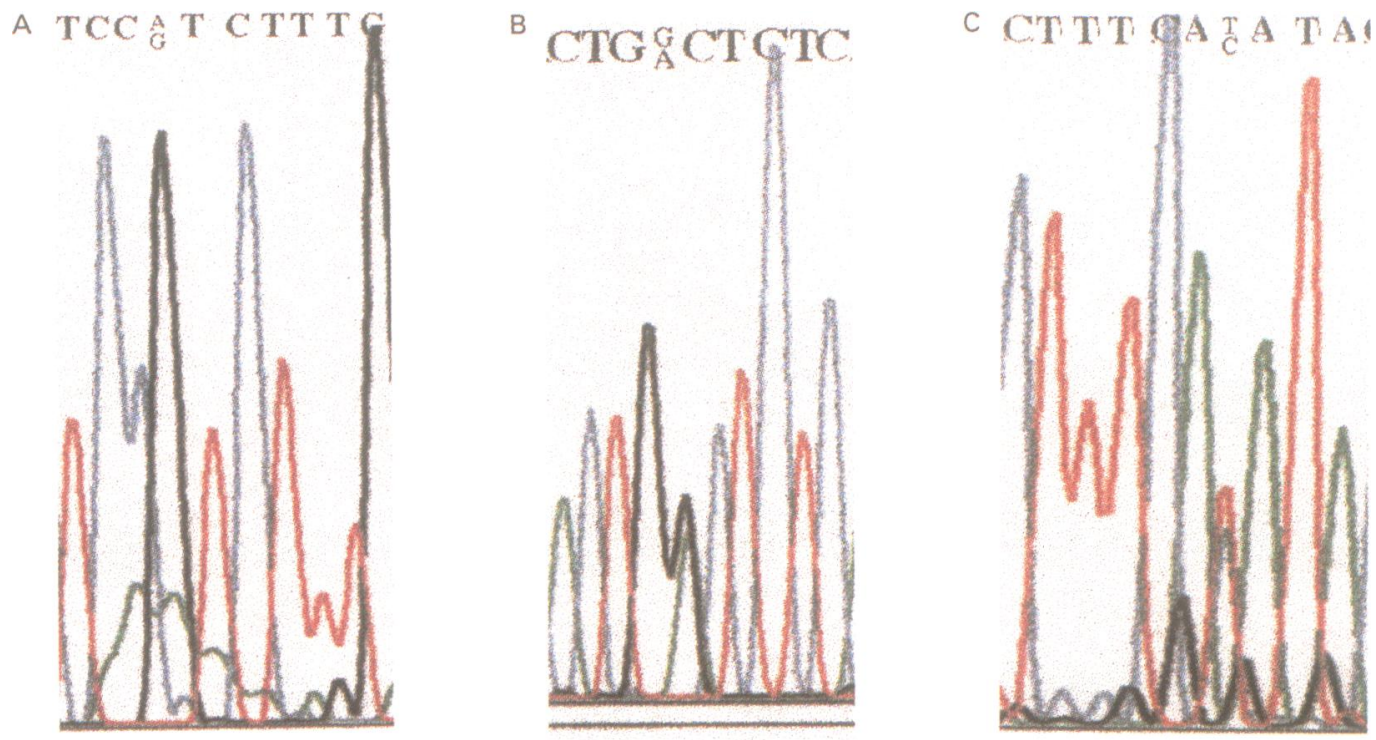

Figure 1 Sequences of germline variants detected (see table 2). (A) Case 78, (B) case $\mathcal{F} M,(C)$ case $Y K Y$.

any first or second degree relative was known to have benign or malignant colorectal tumours, or another HNPCC cancer, or a tumour such as breast cancer that is reportedly associated with colorectal cancer. ${ }^{7}$ Hereditary polyposis syndromes were also excluded. The following clinical data were available: age at presentation, tumour site, and Dukes's stage and grade.

\section{Materials and methods}

Peripheral blood or normal tissue was collected from each patient and DNA was extracted from these samples using standard techniques. Each exon of hMSH2 and hMLH1 (mutations in which account for $>90 \%$ of HNPCC) was amplified specifically using the polymerase chain reaction (for oligonucleotide sequences and conditions, see Beck et al, in preparation; details available from authors). Single strand conformational polymorphism (SSCP) analysis was used to detect mutations, with $10 \%$ acrylamide:bisacrylamide $(30: 0.8)$ gels run at $20 \mathrm{~mA}$ for 18 to 24 hours and products detected by silver staining. For all cases with SSCP band shifts suggestive of mutations, two independent PCR products were sequenced alongside control samples, using the Applied Biosystems (Foster City, California) Ready Reaction Dye Terminator Cycle Sequencing kit and 377 fluorescence based, semiautomated DNA sequencer.

\section{Results and discussion}

Germline HNPCC variants were detected in three of the 50 cases $(6 \%$, table 2 , fig 1$)$.
One of these variants (hMLH1 codon 77 ) is probably a mutation and has not been reported before in colon cancer. The other variants have been reported previously. The hMSH2 codon 322 variant was found by Liu $e t a l^{\beta}$ and Froggatt et $a l^{9}$ and may either be a low penetrance mutation or low frequency polymorphism with normal function. The hMLH1 variant at codon 219 was reported by Liu et $a l^{\beta}$ as a polymorphism with a frequency of $13 \%$, although it is not clear how large a sample was used to determine this frequency. Unless the hMLH1 codon 219 variant actually protects against colon cancer, our data suggest that this allele has a frequency of $1 \%$ at most and we cannot exclude a low penetrance effect on colon cancer risk. It is interesting in this regard that all the variants that we detected were missense changes from the wild type, when an excess of frameshift over missense mutations was expected from HNPCC family data ${ }^{10}$; missense mutations may have less effect on protein function and thus have reduced penetrance. Samples were not available from the parents of any of the three patients with hMSH2 or hMLH1 variants and it was not possible, therefore, to determine whether any of these people had inherited their genotypes or were new mutations. Although too few patients had variant alleles to test formally for associations with the clinical data, none of the three patients had notably extreme clinical features (table 2), such as an exceptionally early age of presentation relative to the other patients studied. Our results suggest that, in the absence of family history, patients with early onset CRC are unlikely to have germline HNPCC mutations. 
Some inaccuracy is inevitable in our estimate of a $6 \%$ (maximum) frequency of germline HNPCC mutations in non-familial CRC which presents at $\leq 45$ years of age. Three features will cause our estimate to be too low. First, we have not studied hPMS1 or hPMS2. Mutations at these loci may be poorly penetrant and more common in apparently sporadic cases of CRC than in HNPCC families, although there is evidence against this suggestion. ${ }^{11}$ Second, SSCP is unable to detect all mutations, ${ }^{12-15}$ with a sensitivity of 70 to $90 \%$ for small scale mutations; SSCP is not suitable, moreover, for detecting the whole exon deletions that have been reported to occur in $\mathrm{hMSH} 2$ and hMLH $1 .^{10}$ Third, some patients may have had a familial disease, such as attenuated APC, ${ }^{1617}$ which went undetected. Two factors may cause our estimate of $6 \%$ to be too high. First, imperfect reporting or recording of family histories may have led some truly familial cases of HNPCC to be included in our sample. Since most patients were ascertained through surgeons with a special interest in colorectal cancer, from dedicated colorectal units, or through cancer genetics clinics, we believe that inaccuracy from this source is probably low. Second, HNPCC may have a better prognosis than other types of colorectal cancer ${ }^{18}$; although not all our patients were alive at the time of this study, it is possible that early death of other cases caused HNPCC to be over-represented in our sample. Taking all factors into account, we suggest that fewer than $10 \%$ of CRC cases under 45 years of age have germline HNPCC mutations.

A previous study ${ }^{11}$ analysed 31 cases of CRC who presented before 35 years of age and did not have a family history fulfilling the "Amsterdam criteria". Of these cases, $58 \%$ of the patients' tumours had microsatellite instability; this is found in about $90 \%$ of HNPCC cancers and 10 to $20 \%$ of sporadic CRCs, but many cancers have microsatellite instability without detectable germline or somatic HNPCC mutations. Twelve patients were analysed for germline mutations at hMSH2, hMLH1, hPMS1, and hPMS 2 , and, of these, five $(42 \%)$ had truncating mutations at hMSH2 or hMLH1; no other germline mutations were detected. In contrast, none of our 12 patients under 35 years old had mutations at hMSH 2 or hMLH1 (although one case was 37 years old). Liu et $a l^{11}$ did not study the frequency of germline HNPCC mutations in patients over 35 years of age, although microsatellite instability occurred in only $17 \%$ of the tumours from their patients in the 35 to 55 age group. The differences between our results and those of Liu et $a l^{11}$ probably arise from the more stringent selection against family history that was used in our study. In addition, the age ranges studied may have led to even greater selection against family history in our sample than in that of Liu et al. ${ }^{11}$ Many unaffected, first degree relatives of patients under 35 years may have germline HNPCC mutations and go on to develop cancer in later life. Since our patients were about 10 years older on average, their parents and sibs would have had a greater chance of developing cancer, with less chance of apparent non-penetrance.

Current colonoscopic screening protocols at St Mark's Hospital include people with a 1 in 10 risk or greater of colon cancer. ${ }^{19}$ The first degree relatives of our patients (taken as a group) would not be included in this screening programme on the basis of germline HNPCC mutations alone, because they would be at a maximum risk of about 1 in 14 . We do not suggest, however, that colonoscopic screening should yet be discontinued for the relatives of early onset, sporadic cases of colon cancer. It remains possible, for example, that some of our sample have an inherited predisposition to CRC that is not caused by HNPCC mutations or by known polyposis syndrome loci. Certainly, the widespread availability of predictive genetic tests will soon allow colonoscopic screening to be targeted at the relatives of patients who do carry germline HNPCC mutations.

Our results suggest that low penetrance or new HNPCC mutations are an uncommon cause of colorectal cancer, even in patients with early onset disease. If new mutations are rare, it follows that people with germline HNPCC mutations have a high fitness. If low penetrance mutations are rare, most HNPCC cancers probably present within families. Our estimate of $10 \%$ germline HNPCC mutations in "sporadic" cases presenting under 45 years old cannot be directly extended to the general population; it is likely, however, that there is a much lower frequency of germline HNPCC mutations among the great majority of sporadic colon cancer patients, who present at over 45 years of age. The estimate that $15 \%$ of all CRCs result from HNPCC is probably much too high. It is unlikely that new or low penetrance HNPCC mutations contribute significantly to the risk of colorectal cancer in the general population. If so, mass population screening for germline defects at HNPCC $\operatorname{loci}^{20}$ may be of relatively little value until technological advances have made the task of mutation detection very much easier.

1 Dunlop MG. Molecular genetics of colon cancer. In: Cowel $\mathrm{JK}$, ed. The molecular genetics of cancer. Oxford: BIOS Scientific Publications, 1995:113-34

2 Fishel R, Lescoe MK, Rao MR, et al. The human mutator gene homolog MSH2 and its association with hereditary nonpolyposis colon cancer. Cell 1993;75:1027-38.

3 Leach FS, Nicolaides NC, Papadopoulos N, et al. Mutations of a mutS homolog in hereditary nonpolyposis colorectal cancer. Cell 1993;75:1215-25.

4 Nicolaides NC, Papadopoulos N, Liu B, et al. Mutations of two PMS homologues in hereditary nonpolyposis colon cancer. Nature 1994;371:75-80.

5 Papadopoulos N, Nicolaides NC, Wei YF, et al. Mutation of a mutL homolog in hereditary colon cancer. Science 1994;263:1625-9.

6 Houlston RS, Murday V, Harocopos C, Williams CB, Slack J. Screening and genetic counselling for relatives of patients with colorectal cancer in a family cancer clinic. BMF 1990 301:366-8.

7 Ford D, Easton DF, Bishop DT, Narod SA, Goldgar DE. Risks of cancer in BRCA1-mutation carriers. Breast Cancer Linkage Consortium. Lancet 1994;343:692-5.

8 Liu B, Nicolaides NC, Markowitz S, et al. Mismatch repair gene defects in sporadic colorectal cancers with microgene defects in sporadic colorectal cancers
satellite instability. Nat Genet 1995;9:48-55.

9 Froggatt NJ, Joyce JA, Evans DGR, et al. MSH2 sequence variations and inherited colorectal-cancer susceptibility. Eur 7 Cancer 1996;32:178.

10 Liu B, Parsons R, Papadopoulos N, et al. Analysis of mismatch repair genes in hereditary non-polyposis colorecta cancer patients. Nat Med 1996;2:169-74. 
11 Liu B, Farrington SM, Petersen GM, et al. Genetic instability occurs in the majority of young patients with colorectal cancer. Nat Med 1995; 1:348-52.

12 Ushijima T, Hosoya Y, Suzuki T, Sofuni T, Sugimura T, Nagao M. A rapid method for detection of mutations in the lacl gene using PCR-single strand conformation polymorphism analysis: demonstration of its high sensi-
tivity. Mutat Res 1995;334:283-92.

13 Ravnik GM, Glavac D, Dean M. Sensitivity of single-strand conformation polymorphism and heteroduplex method for mutation detection in the cystic fibrosis gene. Hum Mol Genet 1994;3:801-7.

14 Vidal PA, Moller DE. Comparative sensitivity of alternative single-strand conformation polymorphism (SSCP) methods. Biotechniques 1994;17:490-2.

15 Sheffield VC, Beck JS, Kwitek AE, Sandstrom DW, Stone EM. The sensitivity of single-strand conformation poly- morphism analysis for the detection of single base substitutions. Genomics 1993;16:325-32.

16 Friedl W, Meuschel S, Caspari R, et al. Attenuated familial adenomatous polyposis due to a mutation in the $3^{\prime}$ part adenomatous polyposis due to a mutation in the $3^{\prime}$ part
of the APC gene. A clue for understanding the function of the APC gene. A clue for understanding the function
of the APC protein. Hum Genet 1996;97:579-84.

17 Spirio L, Olschwang S, Groden J, et al. Alleles of the APC gene: an attenuated form of familial polyposis. Cell 1993;

18 Lynch HT, Smyrk T, Lynch JF. Overview of natural history, pathology, molecular genetics and management of HNPCC (Lynch syndrome). Int $f$ Cancer 1996;69:38-43.

19 Hodgson SV, Bishop DT, Dunlop MG, Evans DG, Northover JM. Suggested screening guidelines for familial colorectal cancer. $\mathcal{f}$ Med Screening 1995;2:45-51.

20 Plummer SJ, Casey G. Are we any closer to genetic testing for common malignancies? Nat Med 1996;2:156-8 